\title{
Bacteriemia by Capnocytophaga spp in patient with chronic limphoblastic leukaemia: a clinical case
}

\section{Maria Teresa Allù}

Laboratorio Analisi chimico-cliniche e microbiologiche Azienda Ospedaliera Ragusa

\section{Bacteriemia by Capnocytophaga spp in patient with chronic limphoblastic leukaemia: a clinical report}

Key words: Bacteriemia, Immunocompromised patient, Capnocytophaga

\section{SUMMARY}

Capnocytophaga spp., capnophilic and Gram-negative fusiform rods with gliding motility, are common inhabitants of the oral cavity. These microorganisms have been implicated as the causative agent of septicaemia in immunocompromised patients and only occasionally in immunocompetent subjects. In this report, a case of septicaemia caused by Capnocytophaga in a neutropenic patient who had stomatitis and pulmonary infiltrates is described. Antibiotic therapy was adjusted according to the susceptibility pattern of the isolate and the patient subsequentlyrecovered.

\section{INTRODUZIONE}

Il genere Capnocytophaga comprende bastoncelli Gram-negativi, fusiformi (di lunghezza 2-4 $\mu \mathrm{m}$ ), a lenta crescita, prevalentemente capnofili, che si muovono "per scivolamento" e fanno parte della normale popolazione microbica del cavo orofaringeo (12). Il genere comprende sette specie ma studi basati sul sequenziamento del 16S rRNA lo hanno diviso in tre gruppi (1): un primo gruppo che comprende le specie $C$. ochracea, $C$. sputige$n a$ e $C$. haemolytica; un secondo gruppo che include le specie $C$. gingivalis e $C$. granulosa; un terzo gruppo che comprende le specie C. canimorsus e $C$. cynodegmi. Le specie $C$. ochracea, C. gingivalis, $C$. granulosa, $C$. sputigena e $C$. haemolytica colonizzano la cavità orale dell'uomo e sono responsabili di batteriemie ed endocarditi secondarie a lesioni della cavità orale in pazienti immunodepressi; eccezionalmente sono stati descritti casi di infezioni disseminate in pazienti immunocompetenti $(12,10)$. Le specie $C$. canimorsus e C. cynodegmi fanno parte della micropopolazione orale dei cani e dei gatti e le infezioni sostenute da queste specie sono in genere associate a morsicature di cani, a graffi da gatti o a contatti stretti con questi animali $(10,5)$. Gli ospiti sensibili presentano condizioni che possono predisporre a siffatte infezioni: epatopatie alcoliche, interventi di splenectomia, malattia di
Hodgkin, fibrosi polmonare, malattie renali, ulcera peptica ed uso di corticosteroidi $(2,3)$. Infezioni da C. canimorsus e C. cynodegmi sono state occasionalmente riscontrate in pazienti immunocompetenti ed in persone che non avevano avuto alcun contatto con cani o altri animali (5). Scopo del lavoro è la descrizione di un caso clinico di batteriemia da Capnocytophaga in un paziente immunodepresso perché affetto da tempo da leucemia linfatica cronica e con recente lesione del cavo orale.

\section{DESCRIZIONE DEL CASO}

Un soggetto di 68 anni di sesso maschile, affetto da leucemia linfoblastica cronica (diagnosticata nel 1994) ed in trattamento chemioterapico, si ricovera presso 1'U.O. di Oncologia per improvvisa iperpiressia. Il paziente riferisce di essere affetto da bronchite da circa 1 mese. All'esame obiettivo presenta febbre elevata $\left(39^{\circ} \mathrm{C}\right)$ ed una evidente stomatite.

Viene instaurata una terapia con levofloxacina $(500 \mathrm{mg} / \mathrm{die} \mathrm{x} 4 \mathrm{gg}$.) e fluconazolo $(0.2 \mathrm{~g} / \mathrm{die}$ $\mathrm{x} 15 \mathrm{gg})$. L'esame emocromocitometrico nel frattempo rileva un conteggio dei globuli bianchi di $6.6 \times 10^{3}$ elementi $/ \mu 1$ con la seguente formula leucocitaria: neutrofili: $7 \%$, linfociti: $87 \%$, eosinofili: 3\%, luc (cellule grandi non colorate, comprendenti linfociti attivati, blasti): $3 \%$; dei globu-

\section{Corresponding author: Maria Teresa Allù}

Laboratorio di Analisi chimico-cliniche e Microbiologiche, Azienda Ospedaliera di Ragusa

P.zza Caduti di Nassirya, I - 97100 Ragusa

Tel.: 0932600148 - Fax: 0932652387 - E-mail: mariateresa.allu@virgilio.it 
li rossi di $2.800 \times 10^{3}$ elementi $/ \mu 1$, con emoglobina di $8.3 \mathrm{~g} / \mathrm{dl}$ ed ematocrito di $24 \%$; delle piastrine di $36 \times 10^{3}$ elementi $/ \mu 1$.

L'RX del torace evidenzia ispessimento del piccolo setto con stria fibrotica a partenza ilare destra per esiti di flogosi e stria di addensamento a sede apicale a sinistra per esiti di tromboembolia.

Vengono eseguite tre emocolture (ciascuna a distanza di 24 ore), utilizzando per ciascun prelievo un flacone per aerobi e uno per anaerobi (Bactec/Alert, bioMérieux). Dopo 48 ore di incubazione nel sistema si positivizza un flacone per anaerobi. Dal flacone positivo si allestisce un vetrino per la colorazione di Gram e delle subcolture su agar sangue e agar cioccolato incubate in aerobiosi e in $\mathrm{CO} 2$ rispettivamente, nonché una subcultura su agar Schaedler incubata in anaerobiosi. Dall'esame batterioscopico diretto si osservano bastocelli Gram-negativi (di cui viene fatta comunicazione immediata al reparto) e, dopo 48 ore di incubazione, si osserva crescita sia sulle piastre di agar-cioccolato e agar-sangue che sulle piastre di agar Schaedler. Sulle colonie vengono eseguiti i seguenti test: ossidasi, catalasi e "cefinase" (per quest'ultimo test è stato utilizzato un dischetto contenente una cefalosporina cromogena). I tre test risultano negativi. L'identificazione biochimica completa viene effettuata utilizzando la galleria Rapid-ID32ANA (bioMérieux).

L'antibiogramma viene eseguito mediante galleria ATB-ANA (bioMérieux), che consente di saggiare la sensibilità agli antibiotici in terreno semisolido in condizioni simile a quelle delle tecniche di riferimento di agar-diluizione, con i breakpoints conformi alle raccomandazioni dell'NCCLS (8). L'identificazione biochimica dà come risultato Capnocytophaga spp ed il ceppo risulta sensibile a penicillina, piperacillina, cefoxitina, imipenem, cefotetan, ticarcillina-clavulanato, amoxicillinaclavulanato, piperacillina + tazobactam e metronidazolo, mentre risulta resistente a clindamicina. Viene instaurata pertanto una nuova terapia antibiotica con piperacillina + tazobactam (e.v.: $9 \mathrm{~g} / \mathrm{die}$ x 11gg). Dopo alcuni giorni il paziente si sfebbra ed un controllo dell'RX torace evidenzia una risoluzione pressochè totale del reperto parenchimale basale destro.

\section{CONCLUSIONI}

I pazienti immunocompromessi con granulocitopenia in seguito a malattie intercorrenti (leucemia mieloblastica, leucemia linfatica acuta, mieloma multiplo, linfoma di Hodgkin, adenocarcinoma) o a chemioterapie citotossiche, sono suscettibili ad episodi di setticemia da Capnocytophaga di solito conseguenti ad infezioni del cavo orale (stomatiti, gengiviti, periodontiti) o ad ulcerazioni orali
(6). Il caso clinico descritto mostra le tipiche caratteristiche predisponenti all'infezione da Capnocytophaga: il paziente è affetto da una severa granulocitopenia per leucemia linfoblastica cronica in trattamento chemioterapico e presenta una infezione del cavo orale (stomatite) che probabilmente rappresenta la via di ingresso del microorganismo nel circolo ematico; le condizioni cliniche del paziente migliorano dopo trattamento antibiotico specifico in seguito al suo isolamento e alla determinazione in vitro della antibiotico - sensibilità.

La patogenesi dell'infezione da Capnocytophaga non è chiara; alcuni studi effettuati su isolati ottenuti da lesioni orali hanno dimostrato che le specie di Capnocytophaga producono aminopeptidasi (9) e questi enzimi possono essere considerati come fattori di virulenza, in quanto svolgono una azione degradativa diretta sui tessuti subgengivali e periodontali. Sia nel caso di malattie periodontali che di setticemia, le specie di Capnocytophaga sono in grado di produrre molecole dializzabili che alterano la morfologia dei neutrofili e ne inibiscono la chemiotassi (11). Altri studi hanno dimostrato che gli isolati di Capnocytophaga dal sangue mostrano una aumentata resistenza all'azione battericida del siero rispetto agli isolati dalle placche gengivali. Una modificazione della struttura lipolisaccaridica della parete cellulare, per effetto di una mutazione, è stata ritenuta essere responsabile dell'aumentata resistenza all'azione battericida del siero e della patogenicità dei microrganismi isolati da sangue (7).

In ogni caso la descrizione del caso è utile per porre maggiore attenzione clinico-diagnostica a siffatte complicanze infettive in soggetti a rischio in quanto immunocompromessi e all'importanza di un attento e responsabile iter operativo microbiologico.

\section{BIBLIOGRAFIA}

1. Ciantar M, Newman HN, Wilson M, Spratt DA. J Clin Microbiol 2005 April; 43(4): 1894-1901.

2. Fumarola D. Increasing evidence for the pathogenic role of DF-2 organisms. Rev Infect Dis 1988; 10: 668.

3. Hicklin H, Verghese A, Alvarez S. Dysgonic fermenter 2 septicemia. Rev Infect Dis 1987; 884-90.

4. Koneman EW, Allen SD, Dowell VR, et al. Miscellaneous and fastidious Gram-negativi bacilli. In Color Atlas and textbook of Diagnostic Microbiology, 1988 pp. 209-30.

5. Lion C, Escade F, Burdin JC. Capnocytophaga canimorsus infectious in humans: review of the literature and cases report. Eur J Epidemiol 1996; 12(5): 521-33.

6. Martino R, Ramila E, Capdevila JA, et al. Bacteriemia caused by Capnocytophaga species in patients with neutropenia and cancer: results of a multicenter study. 
Clin Infec Dis 2001; 33: E20-2.

7. Mc Gowan JE, Del Rio C. Other Gram-negative bacteria. In Mandell Gl, Douglas RG and Bennett JE (eds): Principle and practice of infectious diseases. Third edition Churchill Livingstone, New York, 1990; 1782-93.

8. Methods for antimicrobial susceptibility testing of anaerobic bacteria NCCLS, M11-A5, January 2000.

9. Nakaura M, Slots J. Amminopeptidasi activity of Capnocytophaga. J Periodont Res 1982; 17: 597-603.

10. Pers C, Gahrn-Hansen B, Frederiksen W.
Capnocytophaga canimorsus septicaemia in Denmark, 1982-1995: review of 39 cases Cli Infect Dis 1996; 23(1): 71-5.

11. Shurin SB, Socransky SS, Sweeny E. A neutrophil disorder induced by Capnocytophaga, a dental microorganism. N Engl J Med 1979; 301: 849-854.

12. Timothy F, Murphy, Dennis L. Kosper. En: Harrison, Fauci, Braunwld, Isselbecher, Wilson, Martin editors. Principios de Medicina Interna vol.1(14 ${ }^{\mathrm{a}}$ edizione $)$. Mc - Graw Hill. Interamericana, 2000; 1060-61. 